\title{
Using automated state space planning for effective management of visual information and learner's attention in virtual reality
}

\author{
Author: Opeoluwa Ladeinde \\ Contributors: Mohammad Abdur Razzaque and The Anh Han
}

November 1, 2019

\begin{abstract}
Educational immersive virtual reality is often tasked with minimising distractions for learners and maintaining or signalling their focus to the right areas. Managing location, density and relevancy of visual information in the virtual environment pertain to this. Essentially this problem could be defined as the need of management of cognitive load from the visual information.

To aid in the automated handling of this problem, this study investigates the use of automated state-space planning to model the current "state" of the virtual environment, and determine from a given pool of steps or "actions", a sequence that prioritise minimising cognitive load from visual information through planning the location and density of objects.

This study also investigates modelling the state of what a learner has been informed of and applied. This enables planning to determine when to have the learner relate concepts to existing knowledge for deeper knowledge; planning their generative learning. These states are planned in conjunction with the virtual environment states. The planning is also responsive to identified changes in the learner's deviated attention, or performance with the task. Together it has the potential to minimise the cognitive load from being taught intrinsic information, and minimising extraneous information from the virtual environment.

What was produced currently does not yield many results beyond the method of planning helping the virtual reality applications manage where information appears, but it at least also established a framework for future testing, and improvements to the used methods. This paper provides in more detail, the background for this topic in immersive virtual reality, its significance, the methods used and an evaluation of the method and how further investigations will be continued.
\end{abstract}

Keywords - Immersive Virtual Reality, Signalling, Cognitive Load Theory, Generative Learning, State Space Planning, Planning Domain Definition Language. 


\section{Introduction}

Virtual reality applications continue to be applied in new ways to employ various kinds teaching methods - in training applications, lectures and serious games ${ }^{1}$ As such, there is a large quantity of research on the use of virtual reality applications for education, ranging from comparisons of other educational medif ${ }^{2}$ to studying how to take advantage of benefits specific to virtual reality applications interfaces 3

Past studies have explored how student motivation and assessed knowledge can be influenced by a range of factors, including differing contexts, subject taught, method of using the media, how the media is used in relation to the learning aims. Their implications, as well as a range of factors which draw attention to the way media is used rather than the medium itself, rather than to directly link any potential improvements to student motivation and assessed knowledge to the use of media to meet the learning aims.

It is important to distinguish the media used (virtual reality), from the teaching methods it can be used for. A commonly cited debate was between Richard Clark [3] and Robert Kozma[4] on media influencing learning. A point could be drawn from the former that learning could be executed regardless of media, as the same cognitive processes could be drawn from the learner[3].

As such, a quantity of research studies focus on identifying what within these media can cause learning, and how to optimise learning in these media. This study is in virtual reality applications for a form of education. A specific teaching methods we discuss are on the use of generative learning techniques to build from the learner's existing knowledge to have the reach their learning aims[5]. The use of generative learning techniques has been performed before in immersive virtual reality in Parong's study [1], where the learning outcomes were improved by the learners being asked to summarise what they learnt in writing, as they went through the application. Our approach to using generative learning techniques differs, as it would be to have them process the knowledge by applying it in the virtual environment. Our paper also discusses the management of the learner's cognitive load[6].

A specific challenge this study investigates is the automated managing of the presentation of visual information (which can present intrinsic or extraneous cognitive load) in immersive virtual reality. Figure 1 is an illustration made to provide an example of how learners can process concepts. This study also investigates how learning aims can be automatically ordered and taught to the learners as a new method to optimise their learning in this media.

To perform this, this study is applying the often investigated but rarely applied[7] methods of AI based planning to identify from a set amount of information on the learner, how to teach what they wish to learn from a list of limited actions a given virtual reality application can perform. This would be done through the use of AI based planning ${ }^{4}$ techniques (which will simply be referred to as "planning" henceforth

\footnotetext{
${ }^{1}$ Serious games: Games created with the aim of educating or informing the player

${ }^{2}$ Such as lecture powerpoint presentations[1].

${ }^{3}$ like optimising immersion, visualisation or fidelity of interaction in virtual reality applications $\sqrt[2]{2}$

${ }^{4}$ Planning is a known field, which - when phrased broadly - consists of the use of algorithms to identify a series of needed steps, to reach an identified goal.
} 


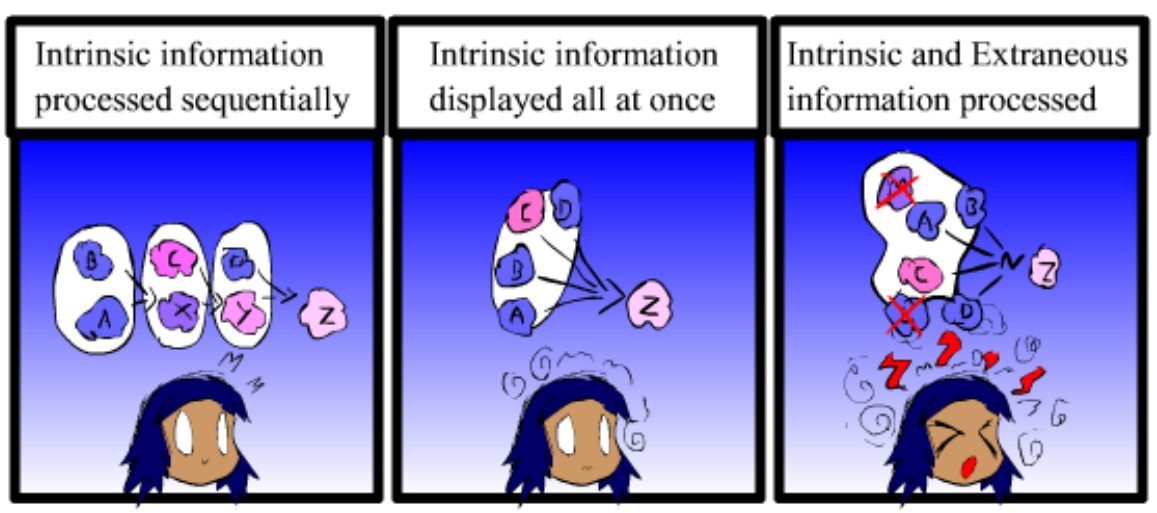

Figure 1: An illustration of processing intrinsic concepts "A", "B", "C" and "D" to meet the aim " $Z$ ". Generative learning techniques would have the tasks get processed and summarised individually and become germane (illustrated as concepts "X" and "Y"), which would be easier to process than all the concepts together. Having to identify what bits of information are relevant to the learner's learning (irrelevant/extraneous information illustrated by "M" and "L") aims increases cognitive load.

in this paper).

Planning can and has been used to have the educational events in (non-immersive) virtual reality applications adapt to be at the level of understanding and comfort of the learner [8][9]. Those studies attempted to use planning to change what happened in the virtual world according to what was expected of the user's engagement.

This study wishes to apply planning to learning in immersive interactive medie ${ }^{5}$ This would involve using planning to help the learner meet learning aims, by using generative learning techniques to create germane information (which could be referred to as "knowledge" in this paper) needed to easily relate or apply deeper learning aims (illustrated in Figure 2). This would also involve identifying the learner's current knowledge on a subject, what in that knowledge has been brought to memory and how to make use of it. This would be done to achieve the learning aims as effectively as possible for the learner from the list of available actions the educational immersive interactive media can do; personalised to what is recorded of the learner's knowledge and preferences.

Ultimately, the study proposed in this paper wishes to investigate how one could use planning for managing the cognitive load from the visualisation of objects, text or information in immersive interactive media - which it currently has not been applied to - as well as using planning for identifying what actions can be given to the user to personalise their experience in this media, to help them reach the learning aims.

This benefits contexts such as this study's case study in radiotherapy treatment. Generally, insufficient health literacy, and hard to process instructions often make pa-

\footnotetext{
${ }^{5}$ interactive media is often tasked with minimising the distractions from its visualisation if it needs the user to focus on a specific area
} 


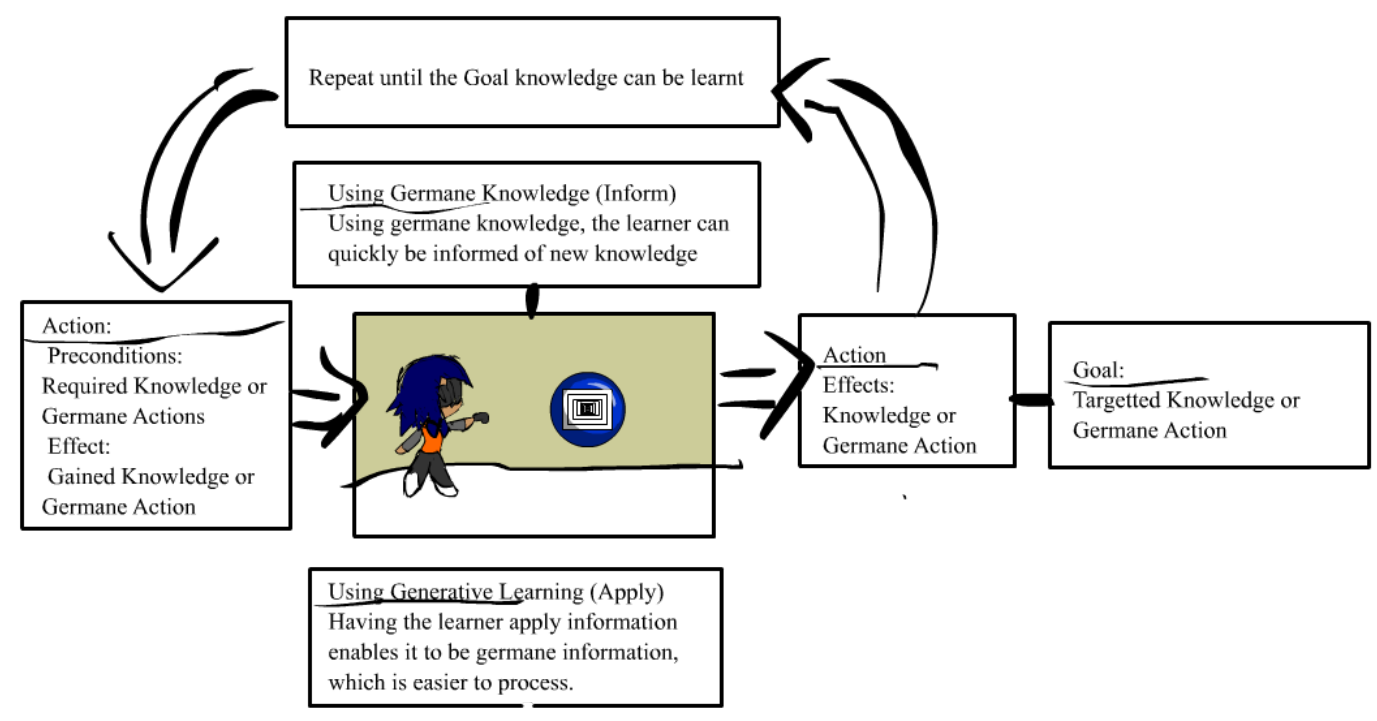

Figure 2: An illustrated diagram on actions informing the learner or having them apply information, to have them able to perform other actions. Also illustrating intrinsic and extrinsic as the relevancy of objects to the action.

tients unable to follow the instructions given by their physician[ [10] [11] [12] [13] [14] [15] [16]. A requirement for radiotherapy treatment for prostate cancer is for patients to have the correct level of bladder filling between their scan and the treatment itself. This is to minimise damage to surrounding organs. Patients often need to have their treatment turned down due to an inability to correctly interpret and follow the given instructions - often choosing not to drink water at all before the treatment [17][18].

For this case, virtual reality applications could be a medium to help patients visualise the effects of their actions on their bladder and the resulting treatment, to inform them of their timing in drinking water, how it changes the position of the organs in the digestive system, and how this would affect the radiotherapy treatment which would inform them on why treatment could need to be deferred, and how to avoid this. Ultimately, using virtual reality for this may have the potential to remove any extrinsic cognitive load from having to understand the written terms in their informative pamphlet, making it easier to process and understand. There would be less of a need for patients to have the higher health literacy to process the instructions (which is still an issue), making them more likely to follow their given instructions.

This case study enables this study to use make use of how immersive virtual reality can help visualise information, but also to use planning around the different levels of health literacy, and present information relative to the understanding of the users. We intend to use planning to construct the events and ways of interacting with the virtual reality application, around the preferences and knowledge of the user, which in this case would be on information pertaining to the effects of bladder filling on their 
radiotherapy treatment, and related knowledge. Using planning would avoid overly simplifying or complicating the information given to the patient's health literacy.

It is important to state that this study does not test in the actual case study itself, but it merely modelled around it. Ultimately, the first goal of this study is to apply state of the art uses of planning into virtual reality applications, to - given a limited pool of learning aims - go through actions to meet those aims in a way preferred and most effective to the learner. The second goal of this study is to adapt the planner to maintain or control the benefits of using virtual reality applications to help a learner visualise information.

\section{Background and Significance}

To reiterate, this paper looks at how we can minimise the cognitive load from the visual information in the virtual world presented by the actions, to reach individual learning aims. This would be in relation to the media being virtual reality, which requires consideration of the location of objects, text, images, and models in 3D space. This would also require consideration of where the user would distribute their attention in this virtual scene.

A large portion of this study is based in planning. There are two key areas that need to be discussed, to provide a scope on what the methods this study wishes to improve upon; the information the planning domains, problems and solutions would need to contain or handle. First we will discuss cognitive load theory (as a more well known example) and how one could apply visualisation techniques in virtual reality while managing the learner's cognitive load. This would then give a scope to our next discussion of uses of planning and how to plan these visualisation techniques.

\subsection{Cognitive load theory}

Cognitive load theory is a relatively well known example for modelling how learners process information[6][19]. This study uses patterns that can be derived from this, as well as similar models (such as Capacity model)[19] for learning in virtual reality. Cognitive load can be phrased as how burdensome processing tasks can become due to related, surrounding or unfamiliar information, that the learner needs to process to accomplish their task.

There are 3 forms of cognitive load. Each of them, and their relation to processed information would be:

- Intrinsic - how demanding tasks that have an identifiable relation to the learner's goal are.

- Extraneous - how demanding restrictions with no direct relation to the learner's goal are, or impositions from the task itself that pose restrictions to how the learner wishes to learn - barriers between learning. For instance, it may be easier for you (the reader) to look at Figure 3 to understand the last sentence (where having to paint a picture from text would be an unneeded step), or that text may be easier for you understand than Figure 3 

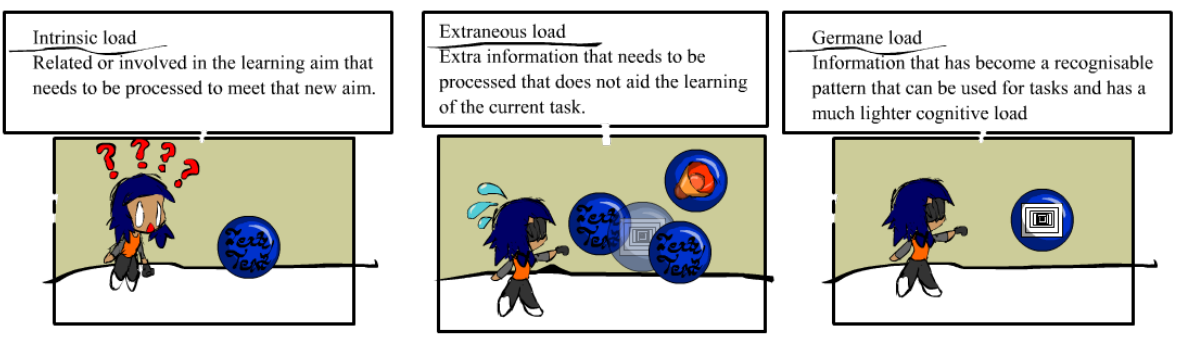

Figure 3: An illustration of what cognitive load is commonly split into, in relation to actions and aims in a virtual environment.

- Germane - relatable experiences, patterned information or actions that reduces the cognitive load, such as using a known methods to perform a task. It is substantially easier to process than identifying or processing given information or a newer concept.

This list is also illustrated in Figure 3

Ultimately, learning can be enhanced by:

- identifying what one could focus on/ do to perform a task successfully[20],

- minimising distractions,

- and the task should use schema, routines or other knowledge familiar to the learner.

This brings importance to managing tasks to be relative to the learner's current understanding. This also requires using information the learner could immediately utilise, apply or study. Managing cognitive load also extends to the presentation of the information given. It also extends to ensuring the relevance of what the user sees; which phrased differently is how information is visualised in immersive interactive media. Virtual reality needs to manage the display of information in 3D space, which provides a large area for distraction or moving relevant information out of view.

Identifying how what can make it easier for users mentally sort through information in $3 \mathrm{D}^{6}$ or how to render information to make it easier to identify key features in $3 \mathrm{D}^{7}$ are techniques that could help with visualising information. This study primarily looks at the aims of actions in the virtual environment, and the location and ordering of them to benefit the learner's focus and learning respectively. This is as opposed to studying the actual techniques involved in the actions themselves (how they visualise) to benefit each task.

Similar to what was identified in Kyritsis' study[21] that identified categorising objects of similar theme together made it easier for the users to identify the needed objects, we will have actions use locations in a way that groups related objects together on request of a given action.

\footnotetext{
${ }^{6}$ like in a study by Kyritsis 21 .

${ }^{7}$ like studies on making it easier to identify features in medical data and biology 22, 23] 25] which relates to our case study
} 


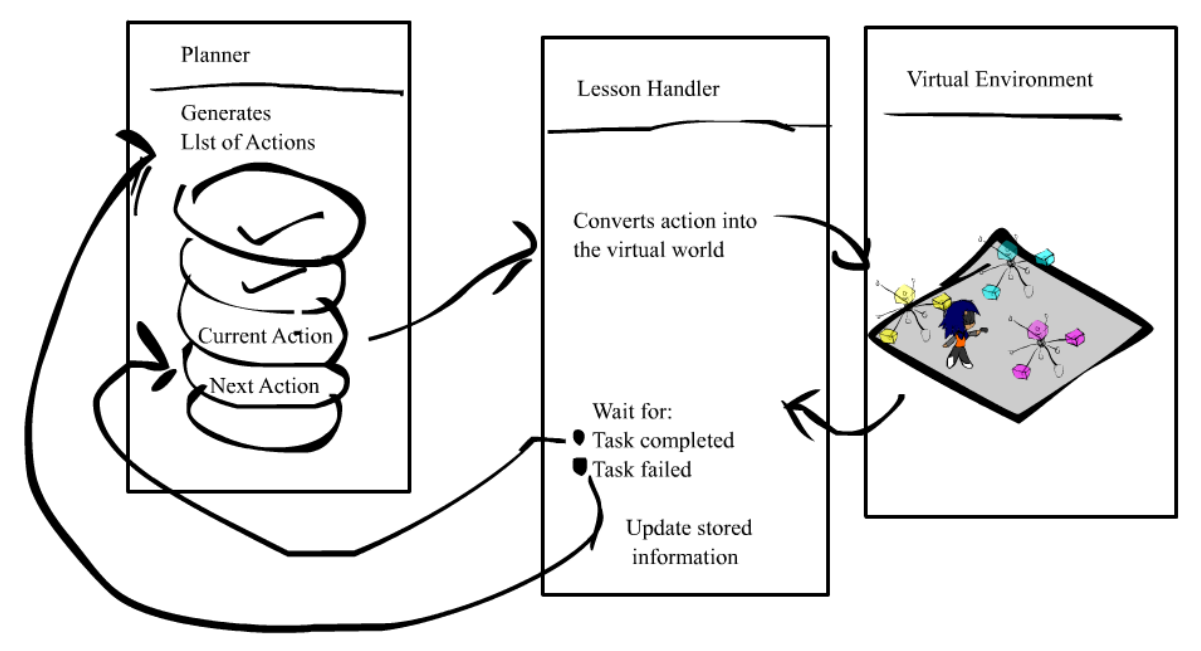

Figure 4: An illustration of how key components of the virtual environment work. The solution, as well as what generates the solution as the "planner". The storing of information of the user, and the state of the world that changes according to changes, or failed plans as well as calls and uses planner solutions called the "Lesson Handler". Finally, what the user sees and interacts with in the "Virtual Environment"

\subsection{A syntactical language for planning}

Planning can be used to identify a sequence of actions needed to reach an aim, given a number of assumptions about the state of the world at the start, and how each action changes the world. There are many applications for planning, in robotics[26], medical treatment plans [27], software applications and more domains it could be applied to[7].

The specific domain this paper will cover is for software applications that use an overarching AI agent to determine what happens in the application. In them, identified plans, or generated solutions are constructed for the agent to use and call the actions accordingly, or call for another plan if the agent believes the plan has failed, or wishes to identify a new goal during the plan. Figure 4 was drawn to help illustrate this.

These particular plans are not intended for the users to see directly. They are only intended to see the result of the plan. However, these plans can still have their intended actions or their reasoning made to be transparent or "explainable" to the user[28]. There are two perspectives this paper identifies: from a user perspective and from a designer perspective.

The aims and direction of the planner's generated plan, as well as why it came to the conclusion this approach would be optimal could be explained to the user. For one, this could potentially improve the trust and motivation of the user to follow the plan, similar to how motivation and matching identified aims has been argued to improve learning outcomes[29].

Another benefit would be to identify and correct what the user believes the plan- 
ner has yet to identify about them, or identified incorrectly about them. The user can inform the planner of any changes to their preferences, or to how they wish to learn. Learning is often stated to require information to flow two ways - to learn about the learner and teach according to that - which this method pertains to.

Ultimately, we'll use the explain-ability of our plans for the user to replan not just on failure, but also on request or an information update. This is in line with one of the aims of the project being to personalise the learning the the learner. It can also theoretically improve the trust from the learner and therefore the learning outcomes, which is a passive benefit that will not be studied in this project, in contrast to the former.

As for explain-ability for domain builders, this would be to make it easier to trace back knowledge or other parameters to an action, or why a given action can not be performed. This would ultimately be for convenience in identifying what to build in the domain, rather than any direct benefit to reaching the learning aims.

This study will also be writing domains and solutions under the Planning Domain Definition Language (PDDL), which standardises planning domains and problems as a modelling language[30][7]. It is one of the oldest conventions for planning, yet has been argued to not have too many researched applied uses, even with tools given and a lot of research on the modelling language itself[7]. Having our study be written under a known convention could provide example uses of PDDL, to influence other project or research uses of PDDL, or vice versa.

This study is working to apply PDDL planning domains, problems and solutions, to plan principals of cognitive load theory, and generative learning into the learning in an immersive interactive education application. The significance of this comes from the use of planning for learning in immersive virtual reality, and to use teaching techniques for using the media to automatically personalise the learning for deeper learning aims. This study wishes to investigate if the theorised benefits of having the planner identify when the learner has been "informed" of something, and when the learner has "applied" something allows it to match the learning in an optimal static learning sequence, while retaining the benefits of personalised learning.

The aims would be structured around having information be immediately relatable to known concepts of the user, based on ideas from making information intrinsic, or to apply known germane information from cognitive load theory (with the latter also relating to generative learning theory). The aims would also be structured around having the user apply the information, based on generative learning techniques. Also the planner will place objects in a way to avoid them being extraneous distractions to the task, and related to related actions.

\section{Method}

In order for it to be intuitive to use a planner to decide the objects that can be created, the information that could be presented or the actions that can be performed, they need to be designed in a way that grants that flexibility in creation, but also interfaces it to the planner at request.

Objects could be considered anything that the user can interact with, that takes 


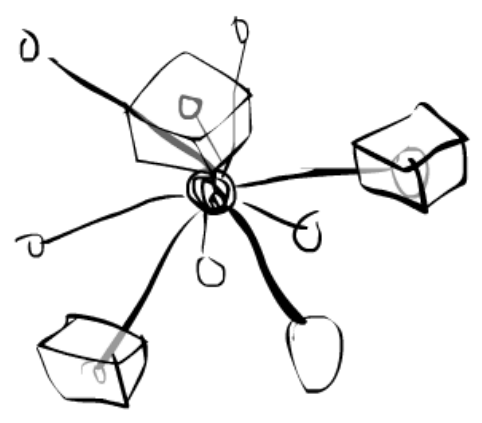

Figure 5: An illustration of an area. The centre of it, as well as empty points objects could appear in are visible. This illustration contains 3 cube shaped objects.

a position in the virtual world and can either be summoned or destroy. For a Unity based project, they could simply be GameObject prefabs (the base structure of an Object that can be copied), and listed referred to by name.

Areas could be considered to be a collection of points in the virtual world that could be identified as the given area with how the points relate to its center illustrated in Figure 5 They would be identified by name.

Knowledge could be considered as any learning aim or skill that would aid or be required to use or relate the relevance of an interface in the virtual world, or needed to be able to relate a future learning aim. This can be information simply told to the learner ("informed"), or the learner summarising what they've learnt by relating it to use in the virtual world ("applied").

Actions could be considered any step that causes a change to the world - the user's knowledge or the objects in the world.

Preferences could be considered any restrictions the user has in interacting with the virtual world (example: absolutely must be sitting at all times), or way the user would prefer to interact with the virtual world (example: physically touch and pick up virtual objects with the remote).

The approach taken in this study was to create a class that facilitates turning existing actions with learning aims, and their associated information, into planning domains. This approach also identifies problems and obtains a solution.

Domains are written specifically for this application (although the structure and use of each term could be reused for any PDDL domain or problem). Although the virtual scene could have created and used an internal planning algorithm, using and writing into a standardised model for planning enables it to be compared to existing models, and its implementation easier to be replicated. With more uses, more techniques as well as problems to improve on are identified, which being under the same convention, helps other uses improve from this. This also lets studies such as this attempt to identify drawbacks and benefits from unique ways of using the conventional language.

One such approach was for the made domains to be dynamic, in terms of what ac- 


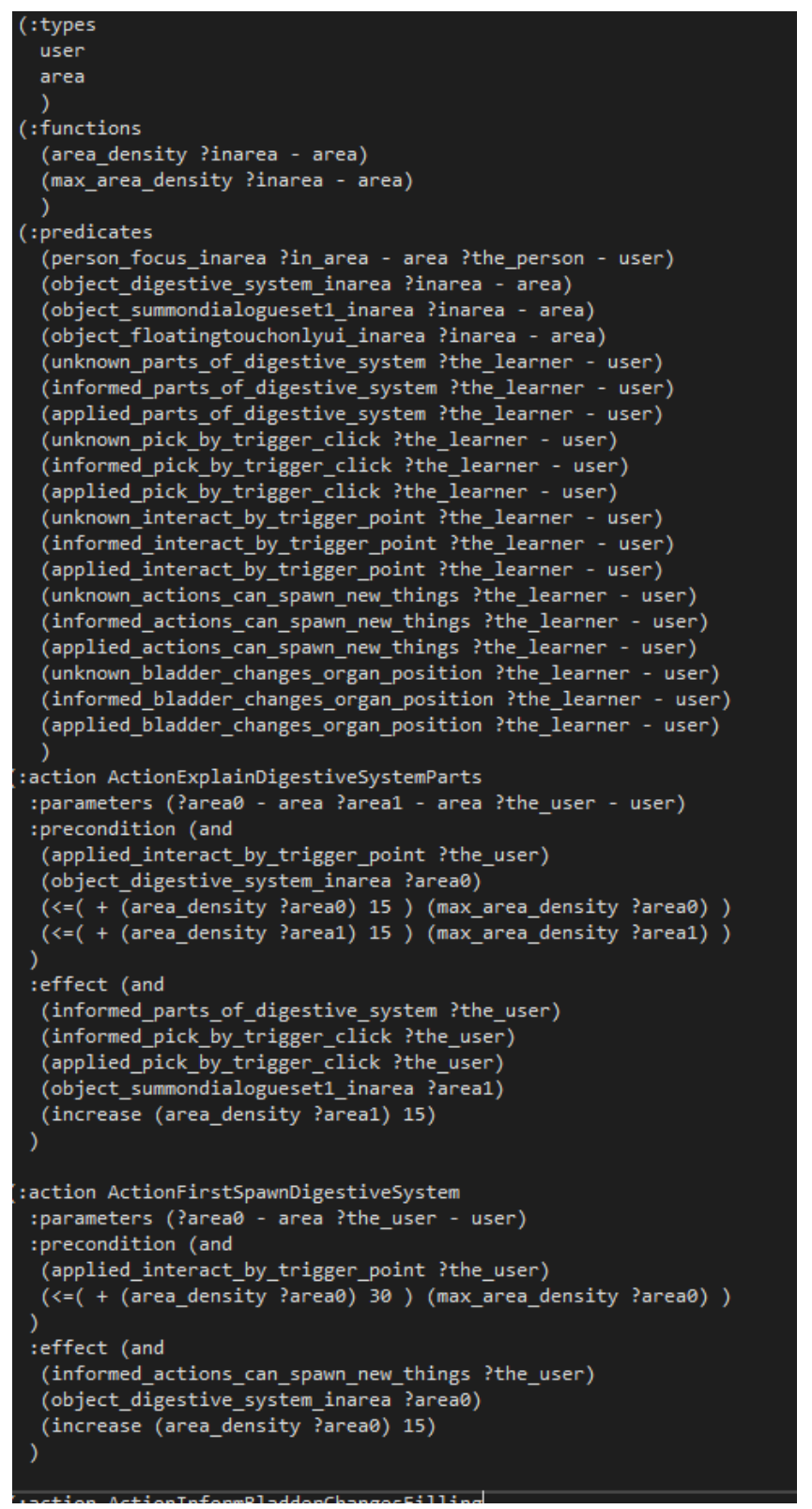

Figure 6: A generated domain. The values "unknown" are not used and will not be in future versions. 


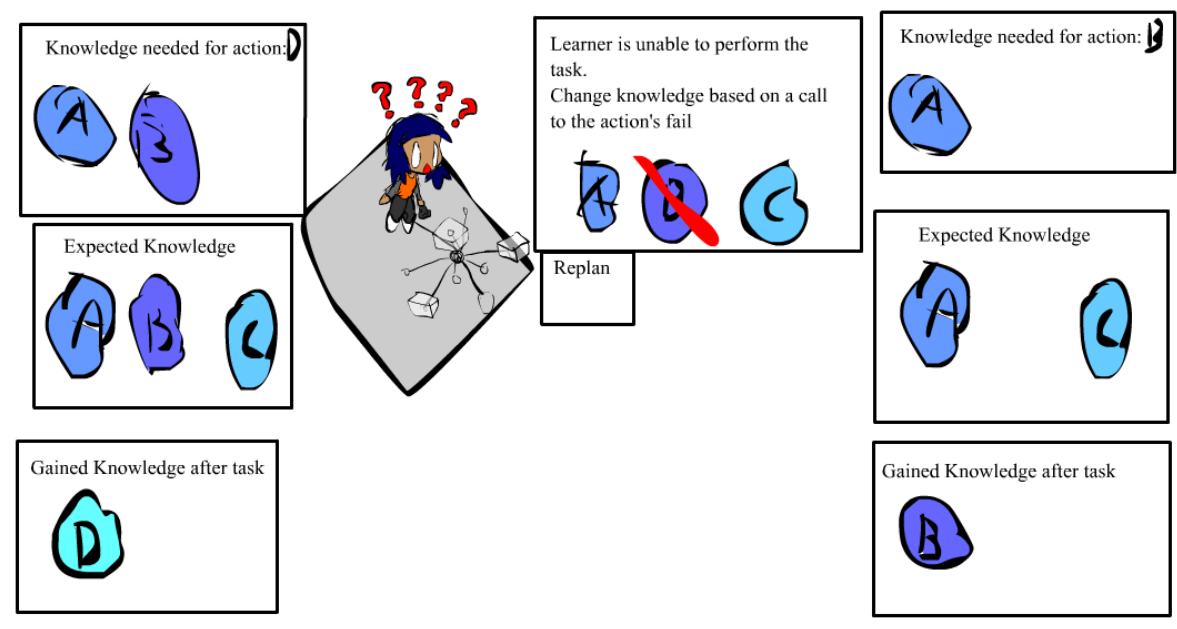

Figure 7: An illustration of the user having difficulty doing the required task, where the planner identifies that the user does not understand concept $B$, so removes that state and re-plans, where the first action is to get the user to understand concept $\mathrm{B}$

tions and knowledge are declared in the domain. The domain changes during the runtime of the application. Even with (relatively at its time) substantially faster planning algorithms like fast-forwards, more actions and conditions slow down how quickly a planner can identify a solution. While it may help this problem, the specific reason this study is taking this approach is actually to shape the solutions the planner finds to be more personalised to the user by using their preferences ${ }^{8}$. We would therefore omit from the domain, actions that do not match the preferences and knowledge that isn't used 9 by any action.

The domains are written as follows. The planner will only be able to plan for factors in the scene that are declared to it.

Areas - Pertains to an in virtual world area, with associated predicates and functions.

User(s) - Pertains to the user with associated predicates and functions.

Actions - Correspond to a possible action and written as actions.

Objects - Built into the domain by predicates. Each have their own density value that is automatically set the change of density in the actions that use them.

Knowledge - Built into the domain by predicates.

In the PDDL domain, the sections are as follows:

• ":types":

\footnotetext{
${ }^{8}$ Personalised learning learns from the learner as they learn, which occurs both before and during learning. Preferences on the other hand are strictly identified before learning begins, and changed and re-planned when their preferences have been identified to be different from what was expected, such as when the user states their preferences have changed

${ }^{9}$ knowledge that is not involved in any action precondition or effect
} 
- "user": Although in the model there is only one, this grants the ability for a planner to identify the focus of multiple users, as well as their knowledge and plan around that.

- "area": An area

• ":functions":

- "max_area_density ?the_area - area": Having this parameter means the planner can identify where objects can be placed, when objects need to be moved or where to shift user attention. This can be the case as long as these rules are attached to the actions.

- "area_density ?the_area - area": A implicit value to refer to how dense a given area is. Areas having an individual density lets them

• ":predicates":

- "person_focus_inarea ?the_area - area ?the_user - user": Having this parameter means the planner can identify where the user is expected to be looking at, and how therefore to know to use an area the user is already looking at, or to either call actions to shift the user's focus to a different area with less density.

- For every object type "object_'name' ?the_area - area": These are automatically laid out in the domain. The main use for this is to be able to create and destroy objects in the planner. In virtual reality, it is possible for objects to be created or destroyed instantly, at any time during the process, as well as multiple of a given object to be created, which is unique to virtual reality. Having them as predicates also enables actions to check for types in a given area, without specifying every object type as a predicate.

- For every knowledge type "informed_'name' ?the_user - user" and "applied_'name' ?the_user - user": These would be the main states for the user in the planner, and there can be multiple. Having them as "informed" and "applied" enables the planner to involve generative learning in the plans, as long as these rules are attached to the actions.

Problems use these same definitions, but naturally identify the current state of the scene (in ':init'), and the knowledge that the user is aiming to obtain (in ':goal').

These domains and problems are automatically created by the AI agents in the virtual world. An example of a generated domain is shown in Figure 6

There may be instances where the user is having difficulty performing a task or performs it with ease. In those instance, the planner would re-plan so the information pertains to them, illustrated in Figure 7 As outlined, the domain contains a parameter for where the learner is expected to focus on. A key time the planner would like re-plan would be when the user has been identified to not be focusing on the area expected, illustrated in Figure 8 


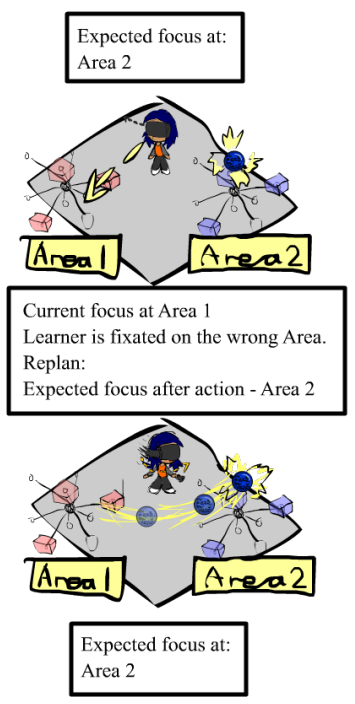

Figure 8: An illustration of the user having their attention identified to be fixating on a different area, and the planner creating a new plan where the first action is to bring the user's attention to the area2.

\subsection{Approach to obtaining results on matching the learning aims}

To reiterate, the study is not on influencing or improving learning using virtual reality, but on how well the uses of stereoscopic view, control and immersive interface in virtual reality can use generative learning techniques, personalised learning and manage cognitive load with the assistance of planning [3].

As such, in order to quantify this we would identify the following in a comparison between two versions of a virtual reality application that makes use of planning, and one that is preset:

- Personalising of the learning to the user. Compared to a version without planning, similar to other studies for the use of planning in a virtual environment [9]. This would have to be identified via a survey.

- Managing the attention of the user. This method should match that of both versions.

What we would identify from our scene would be if planning can successfully perform generative learning relative to the understanding of the learner. Planning has been applied to non-immersive virtual reality before, but the teaching methods of generative learning or management of cognitive load have not been performed in conjunction with stereoscopic visualisation of information.

There are a few challenges in determining the implicit question if planning "works" in virtual reality, or more precisely if it can be used to improve learning in that media.A challenge in identifying where the user is focusing on, is the lack of retina scan. 


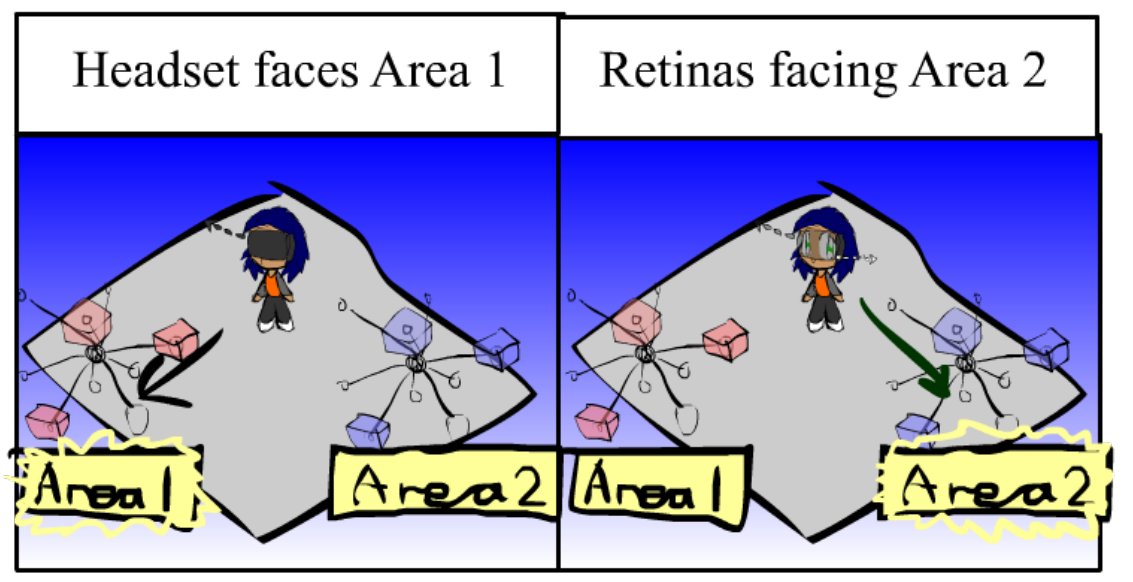

Figure 9: An illustration of the headset facing area 1, yet the user's retinas are looking closer to area 2

The used virtual reality device can identify what direction the headset is facing, which can provide a vague idea on where the user is looking it. However, this may differ from where the use is actually looking (which can be found through retina scans) illustrated by Figure 9 To alleviate this, areas would be spread out so the area of focus could be identified and not be ambiguous.

\section{Conclusion}

Only actions were produced for testing out the plan. There was also little need to involve areas in the usage, but a plan solution could be generated given a problem, and run in the virtual world, using the expected locations. This is similar to the static scripted version of the scene, except any problem could be specified and a plan would be produced and run. For locations, if the user focused on a different area for too long, that would be successfully identified. The virtual scene is shown in Figure 10

As such, it may have greatly helped the study if another case study was chosen, as there would have been a substantially larger amount of actions that could be applied for knowledge and learning aims. Assessing the affects of personalising learning would be long term - over multiple uses - which is both challenging to do, and difficult to compare when most studies are on a singular use when the affects of learning, user motivation and diversity in their knowledge is something that needs to be studies over a long term use. The case study still gave a lot of room for user preferences to be implemented.

Regardless, PDDL was successfully used to apply responsive and personalised learning techniques in an immersive virtual environment. It also at least also established a framework for future testing, and improvements to the used methods by enabling any learning aim to be specified, the current knowledge specified, and the 


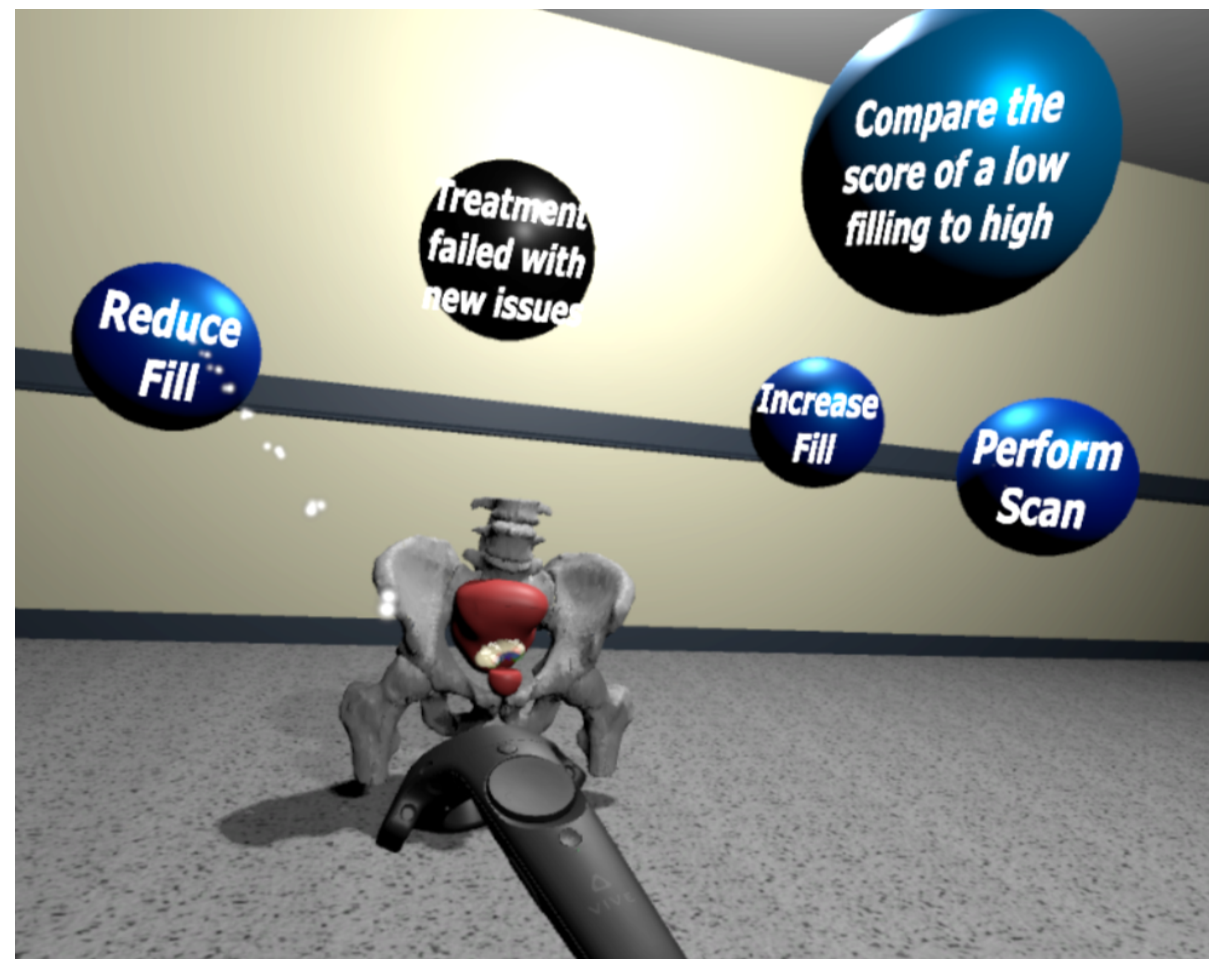

Figure 10: An illustration of the Virtual Scene running 
application would perform the actions to reach those learning aims as optimally for the user as possible.

\section{Acknowledgements}

All authors and contributors are a part of Teesside University. The research is funded by Teesside University.

\section{References}

[1] Jocelyn Paron and Richard Mayer. "Learning Science in Immersive Virtual Reality”. In: Fournal of Educational Psychology (2018).

[2] Fabio Buttussi and Luca Chittaro. "Effects of different types of virtual reality display on presence and learning in a safety training scenario". In: IEEE Transactions on Visualization and Computer Graphics 24.2 (2017).

[3] Richard E Clark. "Media will never influence learning”. In: Educational technology research and development 42.2 (1994), pp. 21-29.

[4] Robert B Kozma. "Will media influence learning? Reframing the debate". In: Educational technology research and development 42.2 (1994), pp. 7-19.

[5] Logan Fiorella and Richard E. Mayer. "Eight Ways to Promote Generative Learning”. In: Educational Psychology Review 28.4 (Dec. 2016), pp. 717-741. Issn: 1573336X. DOI: $10.1007 / \mathrm{s} 10648-015-9348-9$

[6] John Sweller. "CHAPTER TWO - Cognitive Load Theory". In: ed. by Jose P. Mestre and Brian H. Ross. Vol. 55. Psychology of Learning and Motivation. Academic Press, 2011, pp. 37-76. Dor: https : // doi .org/10 .1016/ B978-0-12-387691-1.00002-8

[7] Derek Long, Jan Dolejsi, and Maria Fox. "Building Support for PDDL as a Modelling Tool”. In: KEPS 2018 (), p. 78.

[8] Nahum Alvarez, Antonio Sanchez-Ruiz, Marc Cavazza, Mika Shigematsu, and Helmut Prendinger. "Narrative Balance Management in an Intelligent Biosafety Training Application for Improving User Performance". In: International fournal of Artificial Intelligence in Education 25.1 (2015), pp. 35-59.

[9] Helmut Prendinger, Nahum Alvarez, Antonio Sanchez-Ruiz, Marc Cavazza, joão Catarino, João Oliveira, Rui Prada, Shuji Fujimoto, and Mika Shigematsu. "Intelligent Biohazard Training Based on Real-Time Task Recognition". In: ACM Trans. Interact. Intell. Syst. 6.3 (Sept. 2016), 21:1-21:32. IsSN: 2160-6455. DOI: $10.1145 / 2883617$

[10] Joseph Boyle, Theodore Speroff, Katherine Worley, Aize Cao, Kathryn Goggins, Robert Dittus, and Sunil Kripalani. "Low Health Literacy Is Associated with Increased Transitional Care Needs in Hospitalized Patients”. In: Journal of Hospital Medicine 12 (11 2017), pp. 918-924. 
[11] Laura Cartwright, Levent Dumenci, Brian Cassel, Maria Thomson, and Robin Matsuyama. "Health Literacy Is an Independent Predictor of Cancer Patients' Hospitalizations". In: Health Literacy Research and Practice 1 (1-4 2017).

[12] Rebecca Jessup, Richard Osborne, Alison Beauchamp, Allison Bourne, and Rachelle Buchbinder. "Health literacy of recently hospitalised patients: a cross-sectional survey using the Health Literacy Questionnaire (HLQ)”. In: BMC Health Services Research (2017).

[13] Michael Paasche-Orlow, Ruth Parker, Julie Gazmararian, Lynn Nielsen-Bohlman, and Rima Rudd. "The Prevalence of Limited Health Literacy". In: Journal of General Internal Medicine 20 (2 2005), pp. 175-184.

[14] Richard Safeer and Jann Keenan. "Health Literacy: The Gap Between Physicians and Patients". In: American Family Physician 72 (3 2005), pp. 463-468.

[15] Levent Dumenci, Robin Matsuyama, Daniel Riddle, Laura Cartwright, Robert Perera, Harold Chung, and Laura Siminoff. "Measurement of Cancer Health Literacy and Identification of Patients with Limited Cancer Health Literacy". In: Journal of Health Communication 19 (2 2014), pp. 205-224.

[16] David Breuer and Christophe Lanoux. Health Literacy The Solid Facts. Ed. by Ilona Kickbusch, Pelikan Jürgen, Franklin Apfel, and Agis Tsouros. 2013.

[17] Úna O’Doherty, Helen McNair, Andrew Norman, Elizabeth Miles, Simon Hooper, Mark Davies, Naomi Lincoln, Jan Balyckyi, Peter Childs, David Dearnaley, and Robert Huddart. "Variability of bladder filling in patients receiving radical radiotherapy to the prostate". In: Radiotherapy and Oncology 79.3 (2006), pp. 335340.

[18] Hynds, McGarry, Mitchell, Early, Shum, Stewart, Harney, Cardwell, and O'Sullican. "Assessing the daily consistency of bladder filling using an ultrasonic Bladderscan device in men receiving radical conformal radiotherapy for prostate cancer”. In: 2011 The British Institute of Radiology 84.1005 (2010).

[19] Shalom M Fisch. "Bridging Theory and Practice: Applying Cognitive and Educational Theory to the Design of Educational Media”. In: Cognitive Development in Digital Contexts. Elsevier, 2018, pp. 217-234.

[20] John Sweller. "The worked example effect and human cognition.” In: Learning and instruction (2006).

[21] Markos Kyritsis, Stephen Gulliver, and Eva Feredoes. "Environmental factors and features that influence visual search in a 3D WIMP interface". In: International fournal of Human-Computer Studies 92-93 (2016), pp. 30-43.

[22] Bernhard Preim, Alexandra Baer, Douglas Cunningham, Tobias Isenberg, and Timo Ropinski. "A Survey of Perceptually Motivated 3D Visualization of Medical Image Data”. In: Computer Graphics Forum 35.3 (2016), pp. 501-525.

[23] Ekaterina Prasolova-Forland, Hendrik Hjelle, Hege Tunstad, and Frank Lindseth. "Simulation and visualization of the positioning system of the brain in virtual reality". In: Journal of Computers 12 (3 2017), p. 258. 
[24] Thiago Moraes, Paulo Amorim, Jorge Silva, Silva Pedrini, and Helio Pedrini. "Isosurface rendering of medical images improved by automatic texture mapping". In: Computer Methods in Biomechanics and Biomedical Engineering: Imaging \& Visualization (2016), pp. 1-8.

[25] Yurina Kitaura, Kyoko Hasegawa, Yuichi Sakano, Roberto Lopez-Gulliver, Liang Li, Hiroshi Ando, and Satoshi Tanaka. "Effects of Depth Cues on the Recognition of the Spatial Position of a 3D Object in Transparent Stereoscopic Visualization”. In: International Conference on Innovation in Medicine and Healthcare 71 (2017), pp. 277-282.

[26] Y. Zhang, S. Sreedharan, A. Kulkarni, T. Chakraborti, H. H. Zhuo, and S. Kambhampati. "Plan explicability and predictability for robot task planning". In: 2017 IEEE International Conference on Robotics and Automation (ICRA). May 2017, pp. 1313-1320. DoI:10.1109/ICRA.2017.7989155

[27] Kirsty BradbrookGraham, WinstanleyDavid GlasspoolJohn, and FoxRichard Griffiths. "AI Planning Technology as a Component of Computerised Clinical Practice Guidelines". In: Artificial Intelligence in Medicine (2005), pp. 171-180.

[28] Maria Fox, Derek Long, and Daniele Magazzeni. "Explainable Planning”. In: CoRR abs/1709.10256 (2017).

[29] Jan D. Vermunt and Vincent Donche. "A Learning Patterns Perspective on Student Learning in Higher Education: State of the Art and Moving Forward”. In: Educational Psychology Review 29.2 (June 2017), pp. 269-299. Issn: 1573-336X.

[30] Maria Fox and Derek Long. "PDDL2. 1: An extension to PDDL for expressing temporal planning domains". In: fournal of artificial intelligence research 20 (2003), pp. 61-124. 